\title{
The role of causal connections in the retrieval of text
}

\author{
EDWARD J. O'BRIEN \\ Florida Atlantic University, Boca Raton, Florida \\ and \\ JEROME L. MYERS \\ University of Massachusetts, Amherst, Massachusetts
}

\begin{abstract}
In two experiments, subjects read a series of passages, each containing two target concepts that appeared in widely separated positions within the passage. Following each passage, the time to retrieve each of these concepts was recorded. Several measures from both the Kintsch and van Dijk (1978) model and Trabasso and Sperry's (1985) causal analysis were derived to predict retrieval time. Regression analyses showed that substantial proportions of variance were accounted for by measures derived from a causal analysis. Neither physical position nor measures based on the Kintsch and van Dijk model accounted for significant proportions of variance. Following Experiment 2, a reanalysis of O'Brien's (1987) results revealed that the number of causal connections to an antecedent was a significant predictor of antecedent search time. Results are discussed in terms of a spread of activation through an integrated text representation in which causal connections play a major role.
\end{abstract}

One of the focal points of research in the area of discourse comprehension has been the development of models capable of describing the representation of text in memory (e.g., Grimes, 1975; Johnson-Laird, 1983; Kintsch \& van Dijk, 1978; Mandler \& Johnson, 1977; Rumelhart, 1975; Thorndyke, 1977; van Dijk \& Kintsch, 1983). Although these models differ in many assumptions, they all share the view that the representation is hierarchical in nature. Propositions that reflect important aspects of a text are high in the hierarchy, whereas propositions representing less important details are low in the hierarchy. This distinction has been supported by the consistent finding that important or high-level propositions are more likely to be reported in recall than are less important or low-level propositions (Britton, Meyer, Hodge, \& Glynn, 1980; Britton, Meyer, Simpson, Holdredge, \& Curry, 1979; Cirilo \& Foss, 1980; Kintsch \& Keenan, 1973; Kintsch, Kozminsky, Streby, McKoon, \& Keenan, 1975; Meyer, 1975; Thorndyke, 1977).

Kintsch and van Dijk (1978; van Dijk \& Kintsch, 1983) provided a process model that accounts for these differences in recall as a function of height in a hierarchy. They

This research was supported by NSF Grant BNS83-17900 and NIMH Grant 1R01MH40029-01 awarded to Jerome L. Myers and by NICHD Grant HD17246 awarded to Keith Rayner. We are grateful to Mathew Stanton and Miriam Wade for conducting these experiments and to Jim Chumbley and Chuck Clifton for their many comments during the course of this research. We also would like to thank Howard Hock, Alice Healy, and three anonymous reviewers for their suggestions on improving the manuscript. Requests for reprints should be addressed to Edward J. O'Brien, Department of Psychology, Florida Atlantic University, Boca Raton, FL 33431. proposed that text is processed over a series of cycles with a subset of propositions maintained in short-term memory from one cycle to the next. Probability of recall for any given proposition is considered to be a function of the number of cycles it is maintained in short-term memory. Since the strategy for selecting propositions to be carried over is biased toward higher level propositions, those propositions tend to be maintained in short-term memory longer and therefore have a greater probability of being recalled.

The results of a recent study by O'Brien (1987) are relevant to the assumptions of this hierarchical organization. Subjects read passages in which the final line of each required reinstatement of one of two possible antecedents that had appeared in two widely separated positions in each passage. The passages were constructed using the Kintsch and van Dijk model to ensure that both antecedents were at the same level of the presumed hierarchical representation. O'Brien anticipated that with level held constant, antecedent search time should not be influenced by importance. Averaging over passages, $O$ 'Brien found that search time was a function of distance; the average reinstatement time for the late antecedents was significantly shorter than that for the more distant early antecedents. However, this average effect did not hold for all passages. Importance, as measured by a rating task, was significantly correlated with search time; when a more distan early antecedent was rated as more important than a corresponding late antecedent, the early antecedent was reinstated more quickly. Thus, there appeared to be aspects of importance influencing the search process that were not being captured in a strict hierarchical representation. 
O'Brien (1987) argued that although the basic processing assumptions of the Kintsch and van Dijk model were correct, the accessibility of a proposition was not simply a function of the time it had spent in short-term memory. Instead, he assumed that one consequence of propositions being held in short-term memory is that they become connected to several other propositions. He further assumed that propositions at nonadjacent levels of the Kintsch and van Dijk hierarchy could be connected using both argument overlap and knowledge-based relations. These assumptions lead to a representation consisting of a set of integrated propositions. This representation is similar to that proposed by Anderson $(1976,1983)$, Anderson and Reder (1979), Myers, O'Brien, Balota, and Toyofuku (1984), Trabasso and Sperry (1985), and Trabasso and van den Broek (1985). Trabasso and Sperry presented a particularly detailed depiction of the representation for a sample story.

Within this interconnected representation, important propositions are assumed to differ from less important propositions by the number of interconnections or retrieval routes, rather than by height in the network. This position is similar to that of Trabasso and Sperry (1985) and Trabasso and van den Broek (1985). These authors presented evidence that importance ratings and recall accuracy are directly related to the number of causal paths impinging upon a proposition. Assuming the validity of such an interconnected network, and further assuming that the search through this network is a spread of activation beginning with the currently active propositions, search time also should be related to the network configuration. More precisely, the time to find any particular proposition should be a function of the number of interconnections and of the length of the shortest path from the currently active propositions.

In this study, we examined this predicted relation between search time and causal relations using the stimulus materials from O'Brien (1987). The subjects were cued with a question requiring them to recall aloud either the early or late antecedent in the passage. We used the causal analysis developed by Trabasso and his colleagues (Trabasso \& Sperry, 1985; Trabasso \& van den Broek, 1985) to generate a measure of the number of causal paths to and from the targeted concept and several measures of distance to the targeted concept from various points in the network. These measures then served as predictors of recall time in a multiple regression equation. Other measures, derived from Kintsch and van Dijk's (1978) model of text processing, as well as measures of word length and English-language frequency, also were included in the regression equation.

Our emphasis on causal connections may seem to neglect the role of physical position of a targeted concept within the text. Recall that mean reinstatement times were significantly shorter for late than for early antecedents in O'Brien's (1987) study. This may be because reinstatement took place while subjects were still reading. If so, activation spread not only from nodes in the representa- tion of the antecedent sentence but also from active nodes representing concepts in previously read sentences. The activation from these already active nodes is assumed to produce the position effect that $\mathrm{O}^{\prime} \mathrm{Brien}$ found. However, if retrieval is required after the reading task is completed, there should be no propositions active in memory. Then the time to retrieve a particular proposition should be determined by the pattern of causal connections and should be unaffected by its physical position in the text. We tested position effects by analyzing the results of two manipulations: position of the targeted concept and length of the passage. We also reanalyzed the O'Brien data in terms of Trabasso's causal model to determine whether a different pattern emerges for those reinstatement times than for the recall times of the present experiments.

In summary, the present experiments measured time to retrieve a concept placed either early or late in a previously read passage. Assuming that the recall probe triggers a parallel spread of activation from propositions encountered at the beginning of the story, the length of the shortest causal path from that point to the target should be a major determiner of search time. The number of causal links to and from the target also may play a role. Physical distance to the target should not matter. We also predicted that ratings of the importance of these concepts will be a function of the number of interconnections, as Trabasso and Sperry (1985) and Trabasso and van den Broek (1985) found. Such ratings were obtained by O'Brien (1987) and were reanalyzed in this study.

Although there have been other studies of effects of text structure upon importance and memory, they relied on accuracy measures. Response time should reflect more directly the structures and processes of interest and therefore may well be sensitive to effects not exhibited in accuracy measures.

\section{EXPERIMENT 1}

\section{Method}

Subjects. Forty University of Massachusetts undergraduates participated for course credit. Subjects were randomly assigned to one of four material sets, with the restriction that each set be read by an equal number of subjects.

Materials. The materials were the same 16 passages used by O'Brien (1987). Each passage contained two target concepts; one concept appeared early in the passage and the other occurred late. A sample passage is presented in Table 1. The passages were constructed using the procedures described in Turner and Greene (1978) to ensure control over several factors. First, the early and late concepts were at the same height, or level of importance, in a hierarchical representation. The top-most proposition was selected by using the proposition that resulted in the simplest graph structure (Kintsch \& van Dijk, 1978). Second, to avoid possible primacy effects, 20-40 propositions were placed between the beginning of each passage and the early concept. Also, to eliminate possible recency effects, 20-40 propositions intervened between the late concept and the end of the passage. Two versions of each passage were constructed by varying the number of propositions intervening between the early and late concepts. In the long versions, $40-50$ propositions intervened between the early and late concept. In the short versions, this number was reduced to between 10 and 20 proposi- 
Table 1

Example Passage With Early and Late Concept and Verification Probes*

Cathy decided to go Christmas shopping for her family. She thought that she could find presents for her daughter quickly. Her daughter was three and Cathy knew what to buy her. She had seen an adorable doll and had decided to buy it. However, Cathy had no idea what to get for her husband, and she was getting nervous. [Cathy wondered why she could never find a good present for her husband. When she got to the mall it was crowded and she wanted to leave. She hated shopping when the stores were so crowded. Cathy went to a clothing store and a sporting goods store but she didn't see anything she wanted to buy for her husband.] As she walked by a pet store, she saw a cute puppy. At once she decided that the puppy would be the perfect gift and went in and bought it. [When she left the pet store, she decided to postpone the rest of her shopping until the next day.] While driving home, she passed by a small bakery and she decided to buy a pie. She knew her family would love it. Cathy got back in her car and continued toward her apartment. Suddenly Cathy realized she couldn't keep the puppy in the apartment because she wanted to surprise her husband. Cathy went upstairs to her girlfriend's apartment and asked her friend if she could leave the puppy there until

Christmas. Cathy's friend said yes and invited her inside for coffee.

\section{Verification Probes}

What did Cathy buy her daughter? What did Cathy buy at the bakery? *The early and late concepts are italicized. The short version of this
passage was created by deleting the material between the brackets.

tions. The long versions of the passages ranged in length from 101 to 123 propositions, with a mean of 109 propositions ( 273 words). The short versions contained between 77 and 86 propositions, with a mean of 82 propositions (193 words). The mean placements of the first occurrence of the early and late concepts in the long passages were in propositions 33 and 79 , respectively. In the short versions, the mean first occurrence was in propositions 33 and 48 , respectively. Each passage was followed by two questions; examples are presented in Table 1. Each question required a one-word answer that was either the early or late concept. Four sets of materials were constructed. Within each set, one half of the passages appeared in the long version and the remaining half appeared in the short version. These passages were further subdivided so that for half the passages, the first question required retrieval of the early concept and the second question required retrieval of the late concept. For the other half of the passages, the order of questions was reversed. Each passage appeared an equal number of times in each of these four conditions.

Procedure. Subjects were run individually in an experimental session that lasted approximately $35 \mathrm{~min}$. The passages were displayed on a video terminal controlled by a Zenith Z100 microcomputer. Subjects were instructed to read the passages carefully enough to be able to answer a series of questions that would follow. Each trial began with the word "READY" in the center of the display. When subjects were ready to begin reading a passage, they pressed a line-advance key, which erased the ready signal and presented the first line of the passage. Lines were presented exactly as they appear in Table 1. Subjects were instructed to read each line and to press the line-advance key when they understood it. Each press of the line-advance key erased the current line and presented the next line. Comprehension time for each line was considered to be the time between keypresses. Upon pressing the line-advance key to erase the last line of a passage, the word "QUESTIONS" was presented for $2 \mathrm{sec}$ to inform subjects that the probe sequence was about to begin. Each question was preceded for $500 \mathrm{msec}$ by a ready cue (XXX) that was automatically replaced by the question. The correct answer to each question was always a one-word answer that was either the early or late concept. Subjects were instructed to answer each question out loud, as quickly and as accurately as possible. Their responses triggered a voice key that caused the question to be erased and response time to be recorded. The experimenter provided feedback over an intercom from an adjoining room before presenting the next question. Three practice passages were used to ensure that subjects understood the task and were capable of producing one-word answers both quickly and accurately.

Predictor variables. The recall times were analyzed in regression analyses as a function of 11 predictor variables. Five of these were derived from a causal connections analysis proposed by Trabasso and Sperry (1985) and Trabasso and van den Broek (1985) To obtain these predictors, we divided each passage into a series of idea units using the procedures described by Johnson (1970). Following this, we constructed a causal network for each passage based on the procedures presented by Trabasso and Sperry (1985). To indicate how each of the five predictors based on this analysis were obtained, a simplified causal analysis of a passage is presented in Figure 1. Assume that Idea Units 5 and 10 contain the early and late target concepts, respectively. On the basis of Figure 1, we derived the following measures:

1. CREL. The number of causal relations involving the early and the late target concepts were calculated; this is merely a count of the number of paths to and from an idea unit. CREL is the early minus the late score. In Figure 1, the numbers of connections involving the early and late concepts are two and four, respectively.

2. TOP. This measure reflects the difference in the lengths of the shortest paths from the first idea unit to the early and to the late target concepts. The length of a path was determined by counting the number of intervening causal connections. In Figure 1, the lengths of the shortest paths to the early and late concepts are four and seven, respectively. It is important to note that TOP does not simply reflect the physical positioning of a target concept. For example, if a causal connection existed between Idea Units 2 and 8 (as represented by the dashed line in Figure 1), TOP would be -1 , reflecting a shorter route to the late concept than to the early concept.

3. BOT. This is a measure of the difference in the lengths of the shortest paths from the last idea unit to the early and late target concepts. In Figure 1, the shortest path to the early concept involves six causal connections, whereas for the late concept, there are only two connections.

4. SR. The shortest route to a target concept from either the top or bottom of the causal network was computed. In Figure 1, the shortest route to the early concept begins at the top and involves four connections. The shortest route for the late concept begins at the bottom and involves two connections. SR is the difference in the shortest routes (early - late).

5. SRB. This is a measure of the number of causal connections involved in the shortest route between the two target concepts. In Figure 1, there are four connections on the shortest route between the early and late concepts. 


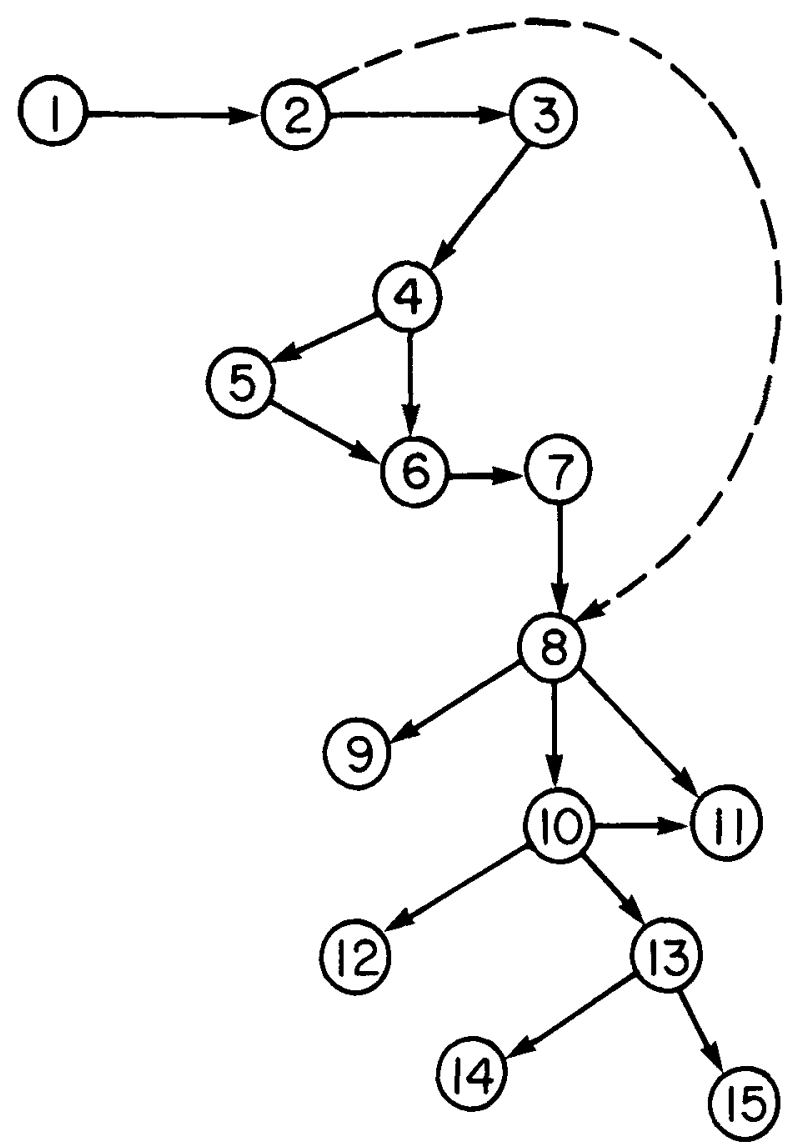

Figure 1. Example of a causal network representation.

As noted earlier, each of the passages was analyzed previously by O'Brien (1987), who used the Kintsch and van Dijk (1978) model. Two additional measures were derived from this analysis. These were:

6. CYC. This is the difference in the number of cycles a target concept spent in the short-term-memory buffer.

7. PROP. This is the number of propositions that shared a connection on the basis of argument overlap with the proposition containing a target concept.

In order to assess whether importance ratings or reading times for the two antecedents captured variance not accounted for by the preceding measures, we added these to our predictor set:

8. IRAT. This is a measure, obtained previously by O'Brien (1987), of the importance of the early and late concepts in the long versions of the passages. The rating task required subjects to decide which of the two concepts was more important to the content of each of the 16 passages. The relative importance of each concept was determined by subtracting the total number of subjects selecting the early concept from those selecting the late concept. Because 40 subjects participated in the rating task, the relative differ- ence in rated importance for the early and late concept could range between -40 and +40 , a positive number suggesting that the early concept was more important and a negative number suggesting that the late concept was more important. The mean difference in rated importance for the 16 passages was $6.81(S D=11.58)$, which did not differ significantly from zero $(p>.5)$.

9. READ. This was simply a measure of the difference in the time to read the line of text containing the early and late target concepts.

Finally, to determine whether differences in the English-language frequency or word length of the early and late concepts were accounting for any variance, the following two measures were included:

10. LFREQ. The difference in the log frequency of the early and late concepts was computed. The raw frequencies were obtained from the Kucera and Francis (1967) norms, with LFREQ $=40$ $+10 \log (f+1)$ (see Chumbley \& Balota, 1984).

11. WL. The difference in the word lengths of the early and late concepts was computed for each passage.

\section{Results}

The data of interest were the mean times to retrieve the early and late concepts. All analyses are based on correct responses only. Retrieval times that were three or more standard deviations from the mean for a subject were eliminated from the analyses. This cut-off procedure eliminated less than $2 \%$ of the scores. Mean response times and percentages of errors for questions pertaining to the early and late concepts are presented in Table 2 .

Analysis of variance. Initial analysis of the retrieval times revealed the following pattern of results. First, there was no difference in the time to retrieve the early or the late target concept $(p>.5)$. Second, subjects required no more time to retrieve a target concept from a long passage than from a short passage $(p>.1)$. The only significant effect was test position. Subjects retrieved a target concept faster when it was in Probe Position 2 than in Probe Position 1. This difference was significant in an analysis based on subject variability $[F(1,36)=5.21$, $p<.05, M S e=38,621]$, but failed to reach significance in an analysis based on item variability $(p>.1)$. There were no reliable differences in an overall analysis of error rates $(p>.5)$.

Regression analysis. The analysis of variance (ANOVA) clearly demonstrates that any differences in retrieval times of early and late concepts were not due to differences in physical distance from the beginning of a passage or to distances between the two targeted concepts. However, there are other variables that we did not manipulate that might have produced differences in retrieval times for early and late concepts. To assess the

Table 2

Mean Retrieval Time and Percentage of Errors (in Parentheses) for the Early and Late Concept as a Function of Passage Length and Probe Position in Experiment 1

\begin{tabular}{lcccccc}
\hline & \multicolumn{2}{c}{ Long Passage } & & \multicolumn{2}{c}{ Short Passage } & \\
\cline { 2 - 3 } Concept & Probe Position 1 & Probe Position 2 & & Probe Position 1 & Probe Position 2 & Mean \\
\hline Early & $1952(.07)$ & $1957(.06)$ & & $1940(.06)$ & $1898(.08)$ & $1937(.07)$ \\
Late & $2024(.06)$ & $1907(.06)$ & & $1948(.08)$ & $1901(.06)$ & $1945(.06)$ \\
\hline
\end{tabular}


effects of these variables, we established 11 predictor variables to be used in a regression analysis. These variables are described in the Method section and summarized in Table 3. Note that CREL, TOP, BOT, SR, and SRB are all based on the causal network analysis of the passages. CYC and PROP are based on O'Brien's (1987) original analysis of the passages using the Kintsch and van Dijk (1978) framework.

These 11 predictor variables were entered into a regression equation, with difference in mean retrieval times for each passage as the dependent variable. The analysis proceeded in two stages. First, a stepwise regression was carried out with a liberal criterion: if a variable's contribution was significant at even the .25 level, it was included in the next stage. The second stage was a regression analysis including all predictors that had met the .25 criterion in the first stage.

Table 4 summarizes the results of this analysis. The variables listed in Column 1 are those that met the .25 criterion in the stepwise regression; they appear in the order in which they were selected. Column 2 presents the proportion of total variance accounted for by the subset of variables defined by a row and all preceding rows. For example, TOP accounts for .392 of the variance, TOP and BOT account for .462 of the variance, and the total subset selected in the stepwise analysis accounts for .833 of the variance. Columns 3 and 4 present the regression coefficients and standard errors for the selected variables. The last column presents the $p$ values (two-tailed) associated with each regression coefficient. For example, after adjusting for the contribution of the other five predictors, the regression of the retrieval time difference upon TOP was significant at the .004 level. Only the four measures of causal distance (TOP, BOT, SR, SRB) even approached significance.

Table 3

Summary of the Predictor Variables Used in Experiments 1 and 2*

$\begin{aligned} & \text { Variable } \\ & \text { CREL }\end{aligned}$ The number of causal connections to and from a concept.
TOP
The length of the shortest route to a concept from the
top of a causal network.
BOT The length of the shortest route to a concept from the
bottom of a causal network.
SR

*Each variable (except SRB) represents a difference score (early - late) for the measure described.
Table 4

Results of Regression Analysis of Retrieval Time Differences in Experiment 1

\begin{tabular}{lccrc}
\hline Variable & $\begin{array}{c}\text { Variance } \\
\text { Accounted For }\left(R^{2}\right)\end{array}$ & $\begin{array}{c}\text { Regression } \\
\text { Coefficient }\end{array}$ & $\begin{array}{c}\text { Standard } \\
\text { Error }\end{array}$ & $p$ Value \\
\hline TOP & .392 & 73.64 & 19.34 & .004 \\
BOT & .462 & -32.14 & 9.39 & .008 \\
SR & .610 & 100.78 & 30.23 & .009 \\
CREL & .746 & -52.02 & 37.96 & .204 \\
SRB & .799 & 33.10 & 15.26 & .058 \\
READ & .833 & -.20 & .15 & .210 \\
\hline
\end{tabular}

It appears from this analysis that variables reflecting factors other than causal distance did not affect the difference in retrieval times between early and late concepts. As a check, however, we entered into a regression equation only the six variables that were not derived from the causal model (CYC, PROP, WL, LFREQ, READ, and IRAT). These variables together accounted for only .224 of the total variance $(F<1)$. In contrast, the five variables derived from Trabasso's causal analysis accounted for .799 of the variance.

\section{Discussion}

The ANOVA revealed only one significant effect. Subjects retrieved a target concept more quickly when it appeared in Probe Position 2 than when it appeared in Probe Position 1. This may be the result of residual activation, following a search for the first probe, facilitating retrieval of the second probe. Alternatively, it may merely reflect that subjects were more set for the second than for the first of two probes of a passage. We conducted Experiment 2 to provide a direct test of these two possibilities.

The fact that there was no difference in the overall times to retrieve an early or a late concept is consistent with the assumption of an interconnected network. The critical determiner of retrieval time should be the number and lengths of paths intersecting a concept. This view finds support in the results of the regression analysis. In particular, differences in retrieval times were highly correlated with differences in the lengths of causal paths leading to the targeted concepts. For example, the greater the difference in causal distance of the antecedents from the first proposition in the story (TOP), the greater the difference in retrieval times.

Before further discussion of these results, validation seems necessary. Regression coefficients are notoriously unstable, particularly with small numbers of data points. Experiment 2 served as a replication of Experiment 1 with one change. To reduce the possibility that there were any propositions active in working memory when the probe was presented, we had the subjects count backward before each recall probe.

\section{EXPERIMENT 2}

\section{Method}

Subjects. Forty University of Massachusents undergraduates participated for course credit. Each subject was randomly assigned to 
a condition, with the restriction that each condition contain an equal number of subjects.

Materials and Procedure. The materials and procedure were the same as in Experiment 1. The only difference was that prior to searching for each target concept, the subjects performed an intervening task. They were presented with a random number between 100 and 999 and counted backward from this number, by $3 \mathrm{~s}$, for $8 \mathrm{sec}$ prior to receiving each question.

\section{Results}

Analysis of variance. The mean times to correctly retrieve the early and late target concepts are presented in Table 5. The same cutoffs were used as in Experiment 1 , resulting in the loss of less than $1 \%$ of the data.

The mean retrieval times paralleled those of Experiment 1 . Subjects retrieved a target concept more quickly when it appeared in Probe Position 2 than when it appeared in Probe Position 1. This difference was reliable in both an analysis based on subject variability $[F(1,36)$ $=7.16, p<.05, M S e=92,436]$ and one based on item variability $[F(1,12)=5.82, p<.05, M S e=64,119]$. There was no difference in the overall times to retrieve an early or a late target concept $(p>.1)$, or in the times to search a long or a short passage $(p>.1)$. In an analysis of error rates, no effects approached significance $(p>.5)$.

Regression analysis. As in Experiment 1, 11 variables (see Table 3) were entered into a stepwise regression analysis as possible predictors of the difference in retrieval times. Table 6 lists the variables that met a .25 criterion for inclusion in the regression equation in the order in which they were selected in the stepwise analysis. Note that the 5 variables derived from Trabasso's causal analysis model were selected first; they were most highly correlated with differences in retrieval time. The four measures of differences in path lengths were all significant at or near the .01 level; some of the difference in retrieval times also was correlated with differences in word frequency and time to read the line of text containing the early and late concepts. As in Experiment 1, variables other than those derived from the causal model did not contribute much total variance. Those 6 variables alone, when entered into a regression equation, accounted for only .386 of the total variance $(F<1)$ when tested against residual variance, whereas the pool of the 5 causally derived variables accounted for .805 of the total variance.

\section{Discussion}

These results are quite consistent with those of Experiment 1 . Once again, the position of and the distance be-
Table 6

Results of Regression Analysis of Retrieval Time Differences in Experiment 2

\begin{tabular}{lcrrr}
\hline Variable & $\begin{array}{c}\text { Variance } \\
\text { Accounted For }\left(R^{2}\right)\end{array}$ & $\begin{array}{c}\text { Regression } \\
\text { Coefficient }\end{array}$ & $\begin{array}{c}\text { Standard } \\
\text { Error }\end{array}$ & $p$ Value \\
\hline TOP & .474 & 48.47 & 12.56 & .006 \\
BOT & .581 & -38.13 & 6.71 & .001 \\
CREL & .680 & -58.93 & 35.45 & .140 \\
SR & .745 & 61.88 & 18.81 & .013 \\
SRB & .805 & 45.67 & 11.29 & .005 \\
LFREQ & .867 & -15.46 & 4.49 & .011 \\
READ & .924 & -.22 & .08 & .038 \\
IRAT & .938 & -4.39 & 3.39 & .237 \\
\hline
\end{tabular}

tween two concepts in a passage had no effect on the speed with which they were recalled. On the other hand, four measures of causal distance between the two concepts accounted for most of the variance in the differences in retrieval times. Indeed, TOP and BOT alone accounted for .581 of the variance. Measures derived from the Kintsch and van Dijk (1978) model made no contribution to the variance even when entered alone into the regression equation. ${ }^{1}$ Even variables such as importance ratings and reading time accounted for little additional variance once the causally derived measures of distance were taken into account.

The probe-position effect found in Experiment 1 remained significant in Experiment 2. Given that subjects in Experiment 2 were required to perform an intervening task between probes, it is unlikely that this effect was due to residual activation that facilitated a second search through the network. Rather, it is more likely that it reflects the fact that subjects were more set for the second probe.

In the two experiments reported so far, the difference in the time to recall the early and late concepts was the dependent variable. Assuming that the subject engages in a top-down search along causal paths, it seems reasonable that measures of causal distance, and particularly TOP, would be important correlates of retrieval time. In contrast, in O'Brien's (1987) earlier study, reading time in response to an anaphoric reference to an antecedent was the dependent variable. In that case, it is less clear that the search would proceed in an orderly fashion from some single point in the causal network. Within a framework such as that of Kintsch and van Dijk (1978), there are concepts from recently read sentences in the propositional buffer. These concepts may represent several points in the network and activation may spread from all these points. Consequently, the results of a regression analysis of the O'Brien data may be somewhat different from those

Table 5

Mean Retrieval Time and Percentage of Errors (in Parentheses) for the Early and Late Concept as a Function of Passage Length and Probe Position in Experiment 2

\begin{tabular}{lcccccc} 
& \multicolumn{2}{c}{ Long Passage } & & \multicolumn{2}{c}{ Short Passage } & \\
\cline { 2 - 3 } \cline { 5 - 6 } Concept & Probe Position 1 & Probe Position 2 & & Probe Position 1 & Probe Position 2 & Mean \\
\hline Early & $1944(.08)$ & $1910(.07)$ & & $1948(.07)$ & $1837(.06)$ & $1910(.07)$ \\
Late & $1947(.06)$ & $1875(.06)$ & & $1981(.07)$ & $1835(.07)$ & $1910(.07)$ \\
\hline
\end{tabular}


reported for the preceding two experiments. In what follows, we report such an analysis of the differences in reinstatement times for early and late antecedents. We also performed a regression analysis of the importance rating data gathered by O'Brien (1987). Trabasso and Sperry (1985) and Trabasso and van den Broek (1985) found that the number of paths intersecting a proposition was an important predictor of rated importance. Our materials are very different from theirs, and we include several predictor variables they did not consider; thus, this analysis provides an opportunity to validate their findings.

\section{REANALYSIS OF O'BRIEN'S (1987) RESULTS}

\section{Method}

In O'Brien (1987), reinstatement times were obtained in the following manner. Subjects read each of the passages using the same procedure described in Experiment 1. The only difference was that an additional sentence was presented that required reinstatement of either the early or the late target concept. As an example, consider the passage in Table 1 . The two target concepts are "doll" and "pie." There were two versions of the final sentence. In one version ("Her friend asked her what she had bought for her daughter"), reinstatement of the early concept "doll" was required. In the other version ("Her friend asked her what she had bought at the bakery"), reinstatement of the late concept "pie" was required. The difference in the times to read these final sentences was used as a measure of the difference in the time to reinstate the appropriate target concept.

\section{Results}

Reinstatement times. The 11 predictor variables (see Table 3) were again entered into a stepwise regression analysis with the difference in reinstatement times as the dependent variable. Only CREL and WL met the .25 criterion for significance in the stepwise regression, and only CREL proved a significant predictor. These results are presented in Table 7.

Importance ratings. The same two-stage analysis was carried out again, but the difference in rated importance of the two target concepts was the dependent variable (and not one of the predictors). Only the four predictors listed in the bottom part of Table 7 contributed variance significant at the .25 level. Note that these are four of the five predictors derived from Trabasso's causal analysis model. Together, they contributed .677 of the total vari-

Table 7

Results of Regression Analysis of Importance Ratings and Reinstatement Time Differences in the O'Brien (1987) Study

\begin{tabular}{lcrrr}
\hline Variable & $\begin{array}{c}\text { Variance } \\
\text { Accounted For }\left(R^{2}\right)\end{array}$ & $\begin{array}{c}\text { Regression } \\
\text { Coefficient }\end{array}$ & $\begin{array}{c}\text { Standard } \\
\text { Error }\end{array}$ & $p$ Value \\
\hline \multicolumn{5}{c}{ Reinstatement Times } \\
CREL & .366 & -159.36 & 59.15 & .018 \\
WL & .431 & -79.03 & 64.97 & .245 \\
\multicolumn{5}{c}{ Importance Ratings } \\
CREL & .370 & 7.94 & 1.81 & .001 \\
SRB & .510 & 1.69 & .77 & .050 \\
TOP & .603 & -1.56 & .94 & .127 \\
BOT & .677 & .61 & .38 & .139 \\
\hline
\end{tabular}

ance of differences in importance ratings. When these four predictors were entered into a regression equation and tested against their standard errors, only CREL clearly made a significant contribution; the SRB regression coefficient was marginally significant. The regression coefficients, their standard errors, and the $p$ values are presented in Table 7 . We also conducted a regression analysis in which the six predictors not derived from the causal model were the only variables entered; these six together accounted for only .139 of the total variance.

\section{Discussion}

Reinstatement times and importance ratings for the early and late antecedents differed largely as a function of the difference in number of intersecting causal paths. The reinstatement time results contrast with those obtained for the recall times analyzed in the present two experiments. The recall times depended primarily on the length of the shortest causal path to a target, whereas the reinstatement times depended on the number of causal paths to and from a target. One possible reason for this difference is that sources of activation are more widespread during reading. Not only is the current input sentence a source of activation, but so also are propositions held in working memory. This situation will produce fast reinstatements when many of these sources are linked to the targeted proposition. In contrast, recall occurs when the short-term propositional buffer is presumably empty. A reasonable strategy then would be to find the beginning of the story and search from there for the required information. We suspect that this search is a parallel spread of activation and, therefore, that the number of causal paths intersecting the target may play some role. However, the most important factor should be the length of the shortest such path.

The importance rating result is consistent with those obtained by Trabasso and Sperry (1985) and Trabasso and van den Broek (1985), who used more traditional stories and a much smaller set of possible predictors. This result is reasonable if we assume that the subject's rating of importance is largely determined by the number of explicit or implicit references to the target. We might view the subject as stepping a counter with each causal path constructed to or from some concept. The final value on that counter is then transformed into an importance rating.

\section{GENERAL DISCUSSION}

One major result of the present experiments is that the mean times to recall concepts that appear early and late in the text do not differ. Also, the physical distance between concepts is unimportant in determining the difference in their recall times. Although acceptance of the null hypothesis is often suspect, these conclusions appear reliable; in neither experiment did the $F$ ratio even approach significance. This result clearly argues against a simple, but plausible, strategy in which subjects attempt to serially recall propositions in the order in which they were 
presented. The result is also consistent with Galambos and Rips's (1982) finding that, even for scripted activities, retrieval time is not influenced by the sequential nature of the material (see also Nottenburg \& Shoben, 1980). This is not to say that serial retrieval based upon input order never occurs. Such an ordered retrieval might occur if subjects were required to recall the entire passage (also, see Barsalou \& Sewel, 1985, who found evidence of sequential effects).

The second major finding is that measures derived from Trabasso's causal analysis of stories were the only significant predictors of recall times in our two experiments, and of importance ratings and antecedent reinstatement times in the study by O'Brien (1987). Measures of differences in lengths of causal naths accounted for substantial and significant port:uls of the variance of differences in recall times for early and late antecedents. Measures of differences in the number of causal connections to the antecedents accounted for much of the variance of differences in importance ratings and reinstatement times. We have argued that recall times reflect a spread of activation down causal paths from the first propositions encountered to the target, with the shortest path dominating the retrieval time. Reinstatement times are measured during reading and reflect activation from many points in the network; therefore, they are more dependent upon the number of paths intersecting with the target. Importance ratings reflect the number of explicit and implicit causal relations into which a proposition entered; to the extent that actions and events cause or are caused by a particular proposition, that proposition is central to the general plan of the story and receives a high importance rating.

Recall accuracy was quite high in this study, but if we had measured it after a more substantial delay, we would have expected it, like importance ratings and reinstatement times, to primarily reflect the number of causal interconnections. The more interconnections, the more retrieval routes there are to a proposition and the more likely that proposition is to be retrieved. Support for this prediction is provided by the analysis by Trabasso and van den Broek (1985), who found that recall probability was a function of number of causal connections. It is possible that path length may also influence recall accuracy. The fewer links in a causal path, the more likely it is that activation will traverse that path. Nevertheless, we would predict that the number of interconnections would be more important than the length of the shortest path to a proposition. This is the opposite of what we found for recall times where path length was considerably more important than number of connections.

Because the early and late targets are at the same level in the Kintsch and van Dijk (1978) text representation, the lack of a difference in retrieval time would be predicted by assuming parallel access within each level of the hierarchical representation. But why should two concepts that are at the same level and accessed in parallel show a systematic difference in retrieval time as a function of the difference in lengths of causal paths to those concepts? Neither the number of cycles during which a proposition was in the short-term buffer (according to the Kintsch and van Dijk model) nor the number of propositions with which its arguments overlapped account for differences in retrieval time. Such differences are addressed by an interconnected representation such as that presented by Trabasso and Sperry (1985) and Trabasso and van den Broek (1985). As they assumed, importance ratings reflect the number of the relations into which propositions enter. Within this framework, effects of levels upon propositional recall (e.g., Britton et al., 1980; Cirilo \& Foss, 1980) occur because higher order propositions have more or stronger connections to other propositions than do lower order propositions. Therefore, there are more retrieval routes to them. However, differences in level are not vital to obtaining effects upon retrieval. Propositions that fall at the same level according to some hierarchical model may well differ in their interconnectedness to other propositions. This is what we presume to have happened in the current experiments.

Fletcher (1986) recently presented another line of evidence of the importance of causal connections. Working within the Kintsch and van Dijk (1978) framework, he compared the ability of eight strategies for allocating short-term-memory capacity to predict verbal protocols and readability. A strategy based on plans and goals of the characters in the texts yielded the best fit of theory to data. As Fletcher noted, this strategy involves the reader in an analysis of causes. Indeed, a count of causal connections based upon the Trabasso representational model led Fletcher to conclude that the success of the plans/goals strategy "seems to reflect the importance of causal connections in this particular set of texts" (Fletcher, 1986, p. 55).

The evidence for the importance of causal connections seems beyond dispute. In addition to the present work and previously cited studies by Trabasso and Sperry (1985), Trabasso and van den Broek (1985), and Fletcher (1986), there are numerous studies evidencing better memory for causally integrated propositions than for temporally sequenced propositions (e.g., Black \& Bern, 1981; Keenan, Baillet, \& Brown, 1984; Myers et al., 1984). What is needed now are studies in which relations among propositions are systematically manipulated while level, as defined within some generally agreed upon text-parsing system, is held constant. Effects of this manipulation on both importance ratings and retrieval times, together with already existing evidence that multiple retrieval routes facilitate memory retrieval (e.g., Myers et al., 1984; Reder, 1980 ) will strengthen the case of an interconnected representation in which causal paths play a major role.

\section{REFERENCES}

ANDERson, J. R. (1976). Language, memory, and thought. Hillsdale, NJ: Erlbaum.

Anderson, J. R. (1983). The architecture of cognition. Cambridge, MA: Harvard University Press. 
Anderson, J. R., \& Reder, L. M. (1979). An elaborative processing explanation of depth of processing. In L. S. Cermak \& F. I. M. Craik (Eds.), Levels of processing in human memory (pp. 385-403). Hillsdale, NJ: Erlbaum.

Barsalou, L. W., \& Sewel, D. R. (1985). Contrasting the representation of scripts and categories. Journal of Memory \& Language, 24 , 646-665.

BLACK, J. B., \& BERN, H. (1981). Causal coherence and memory for events in narratives. Journal of Verbal Leaming \& Verbal Behavior, 20, 267-275

Britton, B. K., Meyer, B. F., Hodge, M. H., \& GlynN, S. M. (1980) Effects of the organization of text on memory: Tests of retrieval and response criterion hypotheses. Joumal of Experimental Psychology: Human Learning \& Memory, 6, 620-629.

Britton, B. K., Meyer, B. F., Simpson, R., Holdredge, T. S., \& CURRY, C. (1979). Effects of the organization of text on memory: Tests of two implications of a selective attention hypothesis. Journal of Experimental Psychology: Human Learning \& Memory, 5, 496-506.

Chumbley, J. I., \& Balota, D. A. (1984). A word's meaning affects the decision in lexical decision. Memory \& Cognition, 12, 590-606.

CiriLo, R. K., \& Foss, D. J. (1980). Text structure and reading times for sentences. Journal of Verbal Learning \& Verbal Behavior, 19 , 96-109.

FletCHER, C. R. (1986). Strategies for allocation of short-term memory during comprehension. Journal of Memory \& Language, 25, 43-58.

Galambos, J. A., \& Rips, L. J. (1982). Memory for routines. Joumal of Verbal Learning \& Verbal Behavior, 21, 260-281

Grimes, J. (1975). The thread of discourse. The Hague: Mouton.

JoHnson, R. E. (1970). Recall of prose as a function of structural importance of the linguistic unit. Journal of Verbal Learning \& Verbal Behavior, 9, 12-20.

Johnson-Laird, P. N. (1983). Mental models. Cambridge, MA: Harvard University Press.

Keenan, J. M., Baillet, S. D., \& Brown, P. (1984). The effects of causal cohesion on comprehension and memory. Journal of Verbal Learning \& Verbal Behavior, 23, 115-126.

KINTSCH, W., \& KEENAN, J. M. (1973). Reading rate as a function of the number of propositions in the base structure of sentences. Cognitive Psychology, 5, 257-274.

Kintsch, W., Kozminsky, E., Streby, W. J., McKoon, G., \& KeEnan, J. M. (1975). Comprehension and recall of text as a function of content variables. Joumal of Verbal Leaming \& Verbal Be havior, 14, 196-214.

KINTSCH, W., \& VAN DIJK, T. A. (1978). Toward a model of text comprehension and production. Psychological Review, 85, 363-394.
KuČera, H., \& Francis, W. (1967), A computational analysis of present day American English. Providence, RI: Brown University Press.

Mandler, J. M., \& Johnson, N. J. (1977). Remembrance of things parsed: Story structure and recall. Cognitive Psychology, 9, 111-191. MEYER, B. (1975). The organization of prose and its effects upon memory. Amsterdam: North-Holland.

Myers, J. L., O'Brien, E. J., Balota, D. A., \& Toyofuku, M. L. (1984). Memory search without interference: The role of integration. Cognitive Psychology, 16, 217-242.

NotTenburg, G., \& Shoben, E. J. (1980). Scripts as linear orders Joumal of Experimental Social Psychology, 16, 329-347.

O'Brien, E. J. (1987). Antecedent search processes and the structure of text. Journal of Experimental Psychology: Learning, Memory \& Cognition, 13, 278-290

REDER, L. M. (1980). The role of elaboration in the comprehension and retention of prose: A critical review. Review of Educational Research, 50, 5-53.

Rumelhart, D. E. (1975). Notes on a schema for stories. In D. G. Bobrow \& A. M. Collins (Eds.), Representation and understanding: Studies in cognitive science (pp. 211-236). New York: Academic Press.

ThORNDYKe, P. W. (1977). Cognitive structures in comprehension and memory of narrative discourse. Cognitive Psychology, 9, 77-110.

Trabasso, T., \& SPERry, L. L. (1985). Causal relatedness and importance of story events. Journal of Memory \& Language, 24, 595-611.

Trabasso, T., \& VAN DEN Broek, P. (1985). Causal thinking and the representation of narrative events. Journal of Memory \& Language, $24,612-630$

Turner, A., \& Greene, E. (1978). The construction of a propositional text base. JSAS Catalog of Selected Documents in Psychology, MS 1713.

Turner, A. A., MCCutchen, D., \& Kintsch, W. (1986, November). The generation of macropropositions during comprehension. Paper presented at the 27th Annual Meeting of the Psychonomic Society, New Orleans, LA

VAN DiJK, T. A., \& KinTsch, W. (1983). Strategies of discourse comprehension. New York: Acadernic Press.

\section{NOTE}

1. Turner, McCutchen, and Kintsch (1986) showed that with relatively long passages, measures derived from the Kintsch and van Dijk (1978) microstructure do not accurately predict recall performance. It is possible that a detailed analysis of the macrostructure would produce results similar to those obtained using Trabasso and Sperry's (1985) causal analysis.

(Manuscript received April 24, 1986 ; revision accepted for publication December 31, 1986.) 